\title{
Intraoperative airway obstruction from a whole dissection of the inner wall of a reinforced endotracheal tube
}

\author{
Eunkyeong Choi ${ }^{1}$, Hae Sun $\mathrm{Cho}^{1}$, and Jong Wha Lee ${ }^{1,2}$ \\ ${ }^{1}$ Department of Anesthesiology and Pain Medicine, Yonsei University College of Medicine, ${ }^{2}$ Division of Anesthesiology and Pain \\ Medicine, Cardiovascular Hospital, Seoul, Korea
}

Modern medical supplies are highly reliable and standardized. Pre-use checklists are definitely in hand, but not thoroughly completed. Here we report a case of a dangerous airway obstruction from a whole dissection of the inner wall of a reinforced endotracheal tube (ETT), which should have been identified prior to intubation.

A 63 -year-old woman $(157 \mathrm{~cm}, 36 \mathrm{~kg})$ was scheduled for a posterior spinal fusion for lumbar spondylolisthesis. Standard monitoring devices, including electrocardiogram lead II, noninvasive blood pressure, pulse oximetry and bispectral index, were applied. After intravenous glycopyrrolate $0.2 \mathrm{mg}$, a remifentanil infusion of $0.1 \mu \mathrm{g} / \mathrm{kg} / \mathrm{min}$ was initiated. Anesthesia was induced with a propofol bolus of $60 \mathrm{mg}$. After confirmation of unconsciousness with loss of verbal response, rocuronium $30 \mathrm{mg}$ was given intravenously for relaxation. One MAC of sevoflurane with $100 \% \mathrm{O}_{2}(>7 \mathrm{~L} / \mathrm{min})$ was given via face mask with manual ventilation. With direct laryngoscopy, endotracheal intubation was done uneventfully with a reinforced ETT (Mallinckrodt ${ }^{\mathrm{TM}}$, Covidien Inc. Mansfield, MA, USA) with an internal diameter (ID) of $6.0 \mathrm{~mm}$ over a metal stylet. On pre-use checking, the tracheal balloon was properly inflated without leaks, and no specific abnormalities were seen. However, metal stylet was managed to be inserted into the lumen with distilled water soaking, which was then attributed to increased resistance from a larger stylet for a smaller ETT. After confirmation of evenly distributed breath sounds on the chest, the ETT was fixed with tape at 20 $\mathrm{cm}$ on the front teeth. Mechanical ventilation was controlled to get an end-tidal $\mathrm{CO}_{2}$ of $35-40 \mathrm{mmHg}$ on the capnography of the anesthesia ventilator (Zeus ${ }^{\circledR}$, Draegerwerk AG \& Co., Lubeck, Germany). Anesthesia was maintained with sevoflurane in a mixture of oxygen and air with $\mathrm{FiO}_{2}$ of 0.6. About $10 \mathrm{~min}$ after intubation, it was noticed that peak inspiratory pressure (PIP) was measured as $>38 \mathrm{cmH}_{2} \mathrm{O}$, and expiratory tidal volume was $75-80 \%$ of the set volume. On the primary suspicion of collapsed lungs, recruitment maneuvers were done manually. Then, the PIP was reduced to $20 \mathrm{cmH}_{2} \mathrm{O}$, but, expiratory tidal volume was still about $50-100 \mathrm{ml}$ less than the inspired volume. To find the leak, the entire circuit was systemically checked, but no specific abnormalities were found. The tracheal balloon was inflated again because the pilot balloon was palpated to be loosened. Immediately after the change to a decubitus position, PIP was again elevated to $41 \mathrm{cmH}_{2} \mathrm{O}$ with expiratory tidal volume of less than $200 \mathrm{ml}$. The patient was immediately returned to the supine position and a pediatric fiberoptic bronchoscope (LF-2, outer diameter of $3.8 \mathrm{~mm}$, Olympus optical Co. Ltd., Tokyo, Japan) was applied under the suspicion of ETT kinking, but failed to be inserted into the ETT. Inevitably, the previous ETT was replaced with a new ETT with an ID of $6.5 \mathrm{~mm}$. The PIP was reduced to $15 \mathrm{cmH}_{2} \mathrm{O}$ with good ventilation. The remainder of the surgical procedure was uneventful, and the patient recovered well without complication. On inspection of the dysfunctional tube, the intraluminal area was shown to be reduced nearly by half with a protruding bulla. For further inspection, the original tube was cut longitudinally along the opposite side of the bulla. It was found that a longitudinal bulla stretched across the entire length, which was a dissection of the plastic film along the inner

Corresponding author: Jong Wha Lee, M.D., Division of Anesthesiology and Pain Medicine, Cardiovascular Hospital, Department of Anesthesiology and Pain Medicine, Yonsei University College of Medicine, 50, Yonsei-ro, Seodaemun-gu, Seoul 120-752, Korea. Tel: 82-2-22288516, Fax: 82-2-364-2951, E-mail: jhanes@yuhs.ac

(C) This is an open-access article distributed under the terms of the Creative Commons Attribution Non-Commercial License (http:// creativecommons.org/licenses/by-nc/3.0/), which permits unrestricted non-commercial use, distribution, and reproduction in any medium, provided the original work is properly cited. 


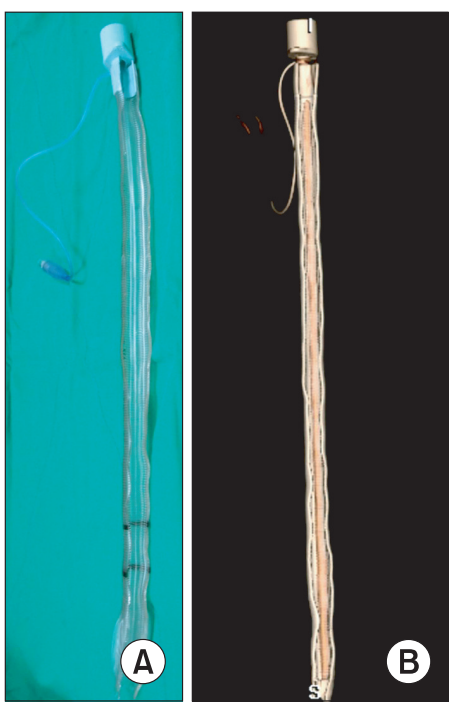

Fig. 1. An external image (A) and 3-dimensional reconstruction of computed tomography (B) of the reinforced endotracheal tube, which was used first in this case, reveal that the tube was cut to show the inner wall bulla, running the whole length of the tube.

wall of the reinforced tube (Fig. 1). This bulla was running along the inner side of the connection between the tracheal and pilot balloon. No evidence of tearing or puncture of the inner side of the tube was found.

Accidental intraoperative airway obstruction related to malfunction of a reinforced ETT has been previously reported [15]. Most of cases resulted from a dissection of the inner wall of a reinforced ETT, which was related to multiple uses after resterilization with an autoclave or ethylene oxide [1,3,4], or to the use of $\mathrm{N}_{2} \mathrm{O}$ during anesthesia [2]. However, the reinforced ETT used in this case was just unpacked before use, and $\mathrm{N}_{2} \mathrm{O}$ was not used. In previous reports, bullae that resulted from a dissection of the inner wall were discrete even when there were multiple bullae. However, the bulla in this case was the whole length of the tube, running on the opposite side of the embedded connection between the tracheal and pilot balloon. The likelihood of a manufacturing error is very high, but cannot be confirmed be- cause the tube was already deconstructed for the full inspection of the inner side.

Setting aside the possibility of manufacturing error, a more detrimental error committed in this case was that no one on the anesthesia team had any suspicion of tube malfunction even when unusual resistance was felt during stylet insertion before use. Because this patient was exceptionally small in build, the tube chosen was smaller than usual. However, a thinner stylet was not readily available. Consequently, it was fairly considered that passing the larger stylet down the smaller tube was difficult. In fact, when a stylet is passed down a reinforced ETT, a certain feeling of resistance is almost always experienced due to the uneven inner surface of a reinforced ETT. Therefore, when resistance was felt in this case, it was attributed to the usual resistance felt during stylet introduction and nobody had a doubt as to tube malfunction. During recruitment maneuvers, the bulla of the inner wall may have been collapsed by the sustained high airway pressure. However, the bulla could re-expand with lowering of the airway pressure. Furthermore, re-inflation of the tracheal balloon done just prior to position change may have worsened the airway obstruction by supplying more air to the bulla. As a result, peak airway pressure was elevated immediately after position change.

In this case, it was fortunate for us to discover the ETT abnormality early before the surgical procedure. While we attribute this to luck, the abnormality should have been discovered before intubation when the unusual resistance was felt with stylet insertion. The lesson in this case is that "vigilance" is not only for monitoring screen, but also for all phases of anesthesia, including medical supplies.

\section{Acknowledgments}

The authors would like to thank Mr. Hyuk Jeon, and Ms. Seon-mi Choi of Cardiovascular intervention laboratory, Cardiovascular Hospital, Yonsei University Health System, who helped the authors to make a 3-dimensional reconstruction of computed tomography images.

\section{References}

1. Paul M, Dueck M, Kampe S, Petzke F. Failure to detect an unusual obstruction in a reinforced endotracheal tube with fiberoptic examination. Anesth Analg 2003; 97: 909-10.

2. Santos IA, Oliveira CA, Ferreira L. Life-threatening ventilatory obstruction due to a defective tracheal tube during spinal surgery in the prone position. Anesthesiology 2005; 103: 214-5.

3. Rao GS, Ali Z, Ramkiran S, Chandrasekhar HS. The dissection of a reinforced endotracheal tube causing near-fatal intraoperative airway obstruction. Anesth Analg 2006; 103: 1624-5.

4. Jeon YS, Kim YS, Joo JD, Kang EG, In JH, Choi JW, et al. Partial airway obstruction caused by dissection of a reinforced endotracheal tube. Eur J Anaesthesiol 2007; 24: 983-4.

5. Rajkumar A, Bajekal R. Intraoperative airway obstruction due to dissection of a reinforced endotracheal tube in a prone patient. J Neurosurg Anesthesiol 2011; 23: 377. 